\title{
A Natural Gel That Relieves Muscle Spasms, Osteoarthritis and Back Pain
}

Received: August 03, 2017; Accepted: August 26, 2017; Published: August 31, 2017

\section{Commentary}

Muscle spasms and sciatica can be extremely painful. Muscle spasms (or cramps) are sudden involuntary contractions of one or more muscles. While they are generally harmless, they can be temporarily debilitating. Osteoarthritis (OA) is the most chronic condition of the joint and the most common type of arthritis and is seen especially among old people. Sciatica, on the other hand, refers to much longer-lasting pain along the sciatic nerve, which branches from the lower back through the hips and down each leg. While self-treatment methods are often effective, severe cases of sciatica may require physical therapy or surgery. Both these conditions may also be treatable with forms of alternative medicine, such as herbal supplements. Emollient Gel is natural product whose anti-inflammatory and antirheumatic activities make it a promising treatment for muscle spasms, osteoarthritis and back pain. Pars Bioscience is pleased to be the first to make Emollient Gel commercially available.

Recurring muscle spasms can result from overuse of the muscle or dehydration. While the pain is sharp, it is not long-lasting. In most cases, these cramps can be self-treated with simple techniques, such as stretching and drinking more water [1]. Osteoarthritis is a type of joint disease that results from breakdown of joint cartilage and underlying bone. The most common symptoms are joint pain and stiffness. Sciatica and other forms of back pain are often caused by a herniated disk or bone spur on the vertebrae. These protrusions can irritate and inflame the sciatic nerve, resulting in acute and persisting pain. When self-treatment methods are not enough, several medications and ointments are available that can reduce the pain. Many of them, like Emollient Gel, are anti-inflammatories [2] (Figure 1).

The strong anti-inflammatory and antirheumatic activities of Emollient Gel are attributable to its phenolic compounds of Curcuma longs and the essential oils of Apium graveolens [3]. These natural components are also responsible for Emollient Gel's anti-inflammatory and analgesic property.

Curcuminoids from $C$. longa exhibit actions at different locations in the pathogenesis of OA once it may play an important role as anti-inflammatory, down-regulating enzymes as phospholipase A2, cyclooxygenase-2 and lipoxygenases. Whereas, celery components showed significant reduction in perceived pain and improved in the functional mobility in patients suffering from

\section{Rafie Hamidpour ${ }^{1,2 *}$ and Luay Rashan²}

1 Department of Herbal Medicine, Pars Bioscience Research Center, Leawood, Kansas, USA

2 Biodiversity Center, Medicinal Plants Division, University of Dhofar, Oman

\section{*Corresponding author: \\ Dr. Rafie Hamidpour \\ ”rafie@parsbioscience.com}

Pars Bioscience Research Center USA, 14109 Cambridge Lane, Leawood, Kansas 66224, USA.

Tel: (913) 432-0107

Fax: (913) 432-5708

Citation: Hamidpour R, Rashan L (2017) A Natural Gel That Relieves Muscle Spasms, Osteoarthritis and Back Pain. Transl Biomed. Vol. 8 No. 3:122

chronic joint, back and muscle pain [4-9]. This gel was tested on more than 1000 patients male and female aged 12 to 81 years old

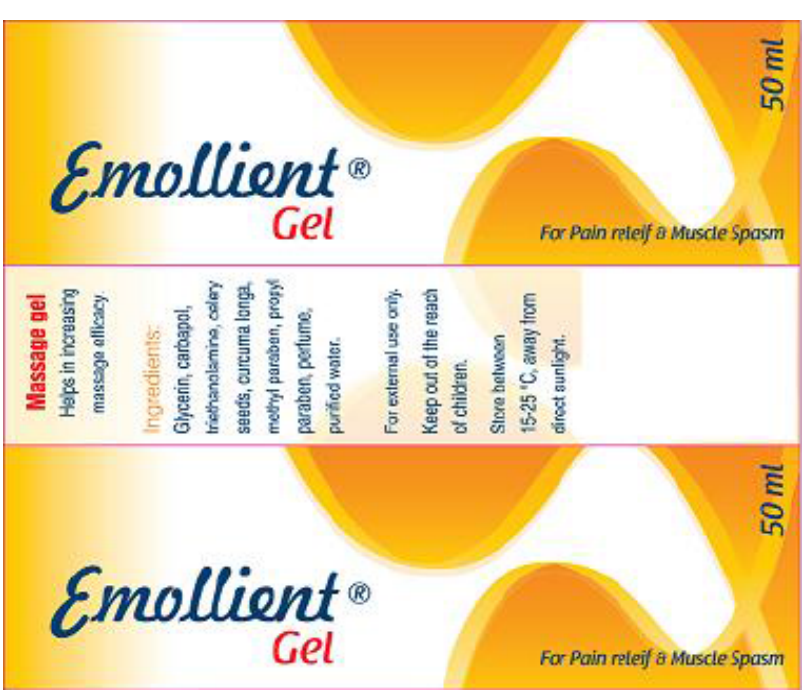

Figure 1 By Pars Bioscience. 
suffering from musculo-cutanous pain associated with injuries such as sport injuries, muscle spasm and pain associated with diseases such as osteoarthritis, muscle spasms, back pain and knee osteoarthritis. These studies have indicated that Emollient Gel can effectively reduce symptoms of muscle spasms and rheumatic diseases such as osteoarthritis and back pain and more than $85 \%$ of these cases showed positive responses. It is very possible, therefore, that this novel natural remedy may be of great use to those suffering from recurring muscle spasms, sciatica, or other forms of back pain [3-5].

Emollient $\mathrm{Gel}$ is a unique natural gel composed of celery seeds and curcuma extracts with different ratios. Celery seeds have potent anti-inflammatory and antirheumatic properties as indicated in the published literatures. On the other hand, curcuma also possesses anti-inflammatory activity because of its role in inhibiting the cyclooxygenase enzyme. Furthermore, Emollient contains other ingredients and some excipients including preservatives [6-9].

\section{Uses of Emollient Gel}

As indicated in both preclinical and clinical studies performed on experimental animals and on many patients suffering from muscle spasms and sciatica-localized rheumatic diseases such as osteoarthritis and back pain, this gel possesses the following characteristics:

\section{References}

1 Mayo Clinic (2016) Muscle cramp. Mayo foundation for medical education and research, USA.

2 Mayo Clinic (2015) Sciatica. Mayo foundation for medical education and research, USA.

3 https://www.niams.nih.gov/

4 Zhang L, Yang Z, Chen D, Huang Z, Li Y, et al. (2017) Variation on composition and bioactivity of essential oils of four common Curcuma herbs. Chem Biodivers.

5 Frank A, Abu-Lafi S, Adawi A, Schwed JS, Stark H, et al. (2017) From medicinal plant extracts to defined chemical compounds targeting
- Exhibited good analgesic property

- Potent anti-inflammatory activity

These properties are attributable to the gel's phenolic compounds, volatile oils such as d-Limonene, essential oils such as Turmerones, and fixed oils such as Eugenol. In addition, it contains some proteins, coumarins, tannins and flavonoids.

How to use emollient gel: Emollient Gel should be applied 3 to 4 times daily to the affected sites and rubbed gently for 2 to 3 minutes in a circular way.

Contra-indications: No contra-indications from using Emollient Gel were reported even if used as adjunct with other pharmaceutical preparations.

Interaction: None reported so far.

Side effects: Emollient Gel is generally well-tolerated. Both preclinical and clinical studies clearly showed that it has no side effects.

Why use natural herbal products:

- Natural

- Safe

- Save money and time

- Help specialists and patients to optimize treatment plan

the histamine $\mathrm{H} 4$ receptor, Curcuma longa in the treatment of inflammation. Inflamm Res.

6 Akuri MC, Barbalho SM, Val RM, Guiguer EL (2017) Reflections about osteoarthritis and curcuma longa. Pharmacogen Rev 11: 8-12.

7 Amalraj A, Pius A, Gopi S, Gopi S (2016) Biological activities of curcuminoids, other biomolecules from turmeric and their derivatives. A review. J Tradit Complement Med 7: 205-233.

8 Dragos D, Gilca M, Gaman L, Vlad A, Losif L, et al. (2017) Phytomedicine in joint disorders. Nutrients.

9 Powanda MC, Whitehouse MW, Rainsford KD (2015) Celery seed and related extracts with antiarthritic, antiulcer and antimicrobial activities. Prog Drug Res 70: 133-135. 